\title{
Confinement losses in microstructured optical fibers
}

\author{
T. P. White, R. C. McPhedran, and C. M. de Sterke \\ School of Physics, University of Sydney, Sydney, NSW 2006, Australia
}

\section{C. Botten}

School of Mathematical Sciences, University of Technology, Sydney, NSW 2008, Australia

\author{
M. J. Steel
}

Department of Applied Physics, Columbia University, 500 West 120th Street, New York, New York 10027

Received May 9, 2001

We describe a multipole formulation that can be used for high-accuracy calculations of the full complex propagation constant of a microstructured optical fiber with a finite number of holes. We show how the imaginary part of the micrastructure, which describes confinement losses not associated with absorption, varies with hole size, the number of rings of holes, and wavelength, and give the minimum number of rings of holes required for a specific loss for given parameters. 2001 Optical Society of America

OCIS codes: $060.2280,060.2400$.

Microstructured optical fibers (MOFs) are one of the most exciting recent developments in fiber optics. A MOF usually consists of a hexagonal arrangement of air holes running down the length of a silica fiber surrounding a central core of solid silica or, in some cases, air. MOFs can exhibit a number of unique properties, including zero dispersion at visible wavelengths and low or high effective nonlinearity. ${ }^{1,2}$ By varying the size of the holes and their number and position, one can also design MOFs with carefully controlled dispersive and modal properties.

In MOFs with solid core regions, the guidance mechanism is somewhat similar to the total internal reflection that occurs in conventional fibers. This analogy is easiest to see at long wavelengths, where the field cannot resolve the individual air holes and the effective cladding index is an appropriate average of air and silica refractive indices. In the modeling of both MOFs and conventional fibers it is usually assumed that the cladding is of infinite extent. ${ }^{3,4}$ Whereas this assumption yields a good approximation for the core modes of conventional fibers, the justification for MOFs has not been established. Issues of this type cannot be studied by use of some existing calculational methods, because these methods have hitherto employed periodic boundary conditions. $^{3-5}$ We recently developed a multipole method that does not use periodic boundary conditions and that is well suited for studying finite cladding effects. $^{6}$ Here we report on confinement losses in MOFs owing to finite numbers of air holes that can cause the modes to be leaky. These losses occur even in the absence of material absorption or scattering losses. Figure 1 illustrates the steady decrease of the Poynting vector with increasing radial distance for a MOF with two rings of holes. Observe that each ring reduces the energy flow by $\sim 1.5 \mathrm{~dB}$, so many rings are required for reduction of the confinement losses to acceptable levels.

We use a full vector multipole formulation in which the modes have generally complex propagation con- stants. $^{6-8}$ The modal fields are expanded in cylindrical harmonic functions about the holes, which are assumed to be circular. This exploitation of the local circular geometry makes the method extremely efficient. In the neighborhood of cylinder $l$, the longitudinal electric field component $E_{z}$ is expanded by use of local polar coordinates $\left(r_{l}, \phi_{l}\right)$ centered on cylinder $l$ as

$$
\begin{aligned}
E_{z}= & \sum_{m=-\infty}^{\infty}\left[a_{m}^{(l)} J_{m}\left(k_{\perp}^{e} r_{l}\right)+b_{m}^{(l)} H_{m}^{(1)}\left(k_{\perp}^{e} r_{l}\right)\right] \\
& \times \exp \left(i m \phi_{l}\right) \exp (i \beta z),
\end{aligned}
$$

where $k_{\perp}^{e}=\left(k_{0}^{2} n_{e}^{2}-\beta^{2}\right)^{1 / 2}$ is the (possibly complex) transverse wave number in the silica background of refractive index $n_{e} ; \beta$ is the mode's propagation constant, which is related to its effective index by $\beta=N k_{0}$, where $k_{0}=2 \pi / \lambda$ is the free-space wave number and $\lambda$

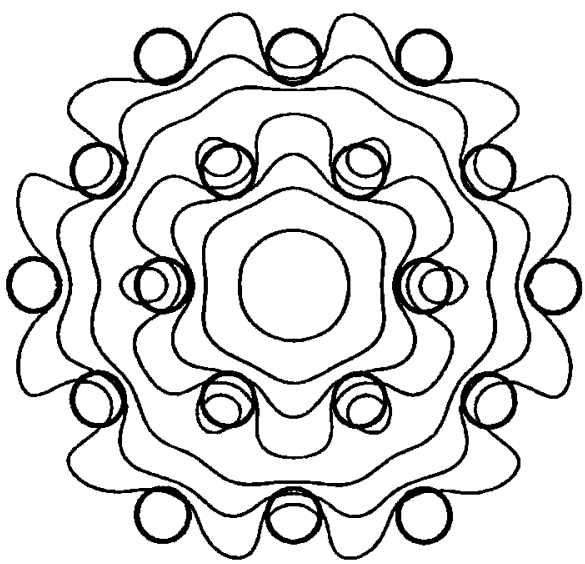

Fig. 1. Longitudinal component of the Poynting vector of the leaky fundamental mode of the MOF consisting of 18 holes of diameter $d=0.92 \mu \mathrm{m}$ with spacing $\Lambda=2.3 \mu \mathrm{m}$. At $\lambda=1.55 \mu \mathrm{m}$ the structural loss of this mode is $1600 \mathrm{~dB} / \mathrm{m}$. Contour lines are at $0.5-\mathrm{dB}$ intervals. 
is the wavelength. A time dependence of $\exp (-i \omega t)$ is assumed. Further, inside cylinder $l$, where the refractive index is $n_{i}=1$, the field is expressed in regular Bessel functions as

$$
E_{z}=\sum_{m=-\infty}^{\infty} c_{m}^{(l)} J_{m}\left(k_{\perp}^{i} r_{l}\right) \exp (i m \phi t) \exp (i \beta z)
$$

where $k_{\perp}^{e}=\left(k_{0}^{2} n_{e}^{2}-\beta^{2}\right)^{1 / 2}$. Similar expansions are used for $H_{z}$. By applying electric and magnetic boundary conditions on the holes' surfaces, we obtain expressions that relate $a_{m}^{(l)}, b_{m}^{(l)}$, and $c_{m}^{(l)}$ on that cylinder. We determine the values of the coefficients for the cylinders collectively by observing that the regular $\left(J_{m}\right)$ part of the field close to cylinder $l$ must be due to the outgoing $\left[H_{m}^{(1)}\right]$ part of the wave field from all other cylinders $j \neq l .^{7}$ Mathematically, we derived these values by using Grafs addition theorem, ${ }^{9}$ which resulted in a relation among the coefficients of the different cylinders. This relation then leads to an infinite set of homogeneous algebraic equations, depending on $\beta$, that can be truncated such that $-M \leq m \leq M$ in Eqs. (1) and (2) and solved numerically.

Surrounding all holes is a jacketing material with complex refractive index $n_{J}=n_{e}+i \delta$, where $\delta \ll 1$, ensuring that the fields and their energy density are integrable in the transverse plane. Without the jacket, the expansions lead to fields that diverge far away from the core, because the modes are not completely bound. Although the jacket is included for mathematical rigor, the effects of its radius $R_{J}$ and $\delta$ on the propagation constants were found to be negligible over a wide range of parameters, provided that the mode was well confined. As an example, for the second-order mode of a six-hole MOF with $n_{e}=1.45$, hole diameter $d=5 \mu \mathrm{m}$, and hole spacing of $\Lambda=6.75 \mu \mathrm{m}, N=1.436773531+i 1.897 \times 10^{-6}$ at $\lambda=1.55 \mu \mathrm{m}$ with $R_{J}=12.25 \mu \mathrm{m}$ and $\delta=1 \times 10^{-4}$, whereas $N=1.436773535+i 1.899 \times 10^{-6}$ with $R_{J}=12.25 \mu \mathrm{m}$ and $\delta=1 \times 10^{-12}$. Changing $R_{J}$ to $20.00 \mu \mathrm{m}$ does not affect $N$ within the accuracy shown. These results were obtained with $M=7$; larger values for $M$ do not change the results within the decimal places quoted.

Our method was tested rigorously for internal consistency and by comparison with results of a beam propagation method (BPM) from a commercial beam propagation package for a simple six-hole MOF structure. In Fig. 2 we show the real and the imaginary parts of the effective index of the fundamental mode for a six-holed MOF with $n_{e}=1.45$, a hole spacing of $4 \mu \mathrm{m}$, and $d=2.26 \mu \mathrm{m}$. There is clearly good agreement between the multipole method and the vector BPM in both the real and the imaginary parts of $N$. We note that the scalar BPM gives results for $N_{r}$ that agree to three decimal places with the vector BPM, though $N_{i}$ has a relative error of $35 \%$ at long wavelengths.

In Fig. 3 we show the confinement loss of a typical MOF as a function of hole size and number of holes. The hole spacing, $\Lambda=2.3 \mu \mathrm{m}$, and the wavelength, $\lambda=1.55 \mu \mathrm{m}$, are both fixed. The confinement loss of the fundamental mode is plotted as a function of the ratio $d / \Lambda$ for one to eight rings of holes (Fig. 3 , inset). The refractive index $n_{e}=1.44402$ was obtained from the Sellmeier equation for silica. ${ }^{10}$ Note that, for small holes and few rings, the losses are huge but decrease rapidly as the holes are enlarged or more rings of holes are introduced. The increase of the loss with decreasing hole size is easy to understand because the lowindex holes provide the confinement mechanism: In the absence of holes, the light is not confined at all. For $d / \Lambda<0.3$, where MOFs are single moded, ${ }^{11}$ eight rings of holes are just sufficient for reaching a benchmark loss value of $1 \mathrm{~dB} / \mathrm{m}$, with a loss of $0.5 \mathrm{~dB} / \mathrm{m}$ at $d / \Lambda=0.3$. Of course, material absorption and fabrication defects contribute to the loss in ways not considered here, but Fig. 3 gives a useful lower bound.

The wavelength dependence of the confinement losses is shown in Fig. 4 for a number of values of

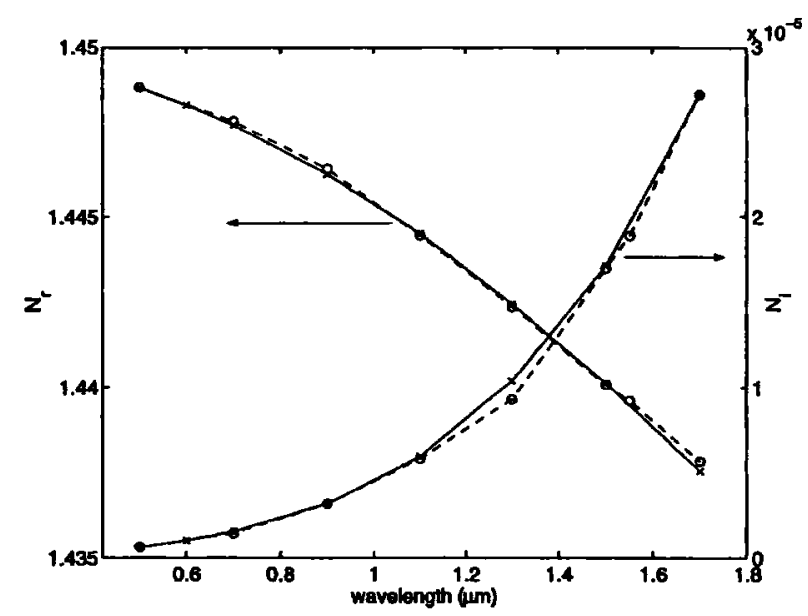

Fig. 2. Comparison of real and imaginary parts of the effective index from the multipole method (crosses) and the vector beam propagation method (circles). The curves were added to aid the eye.

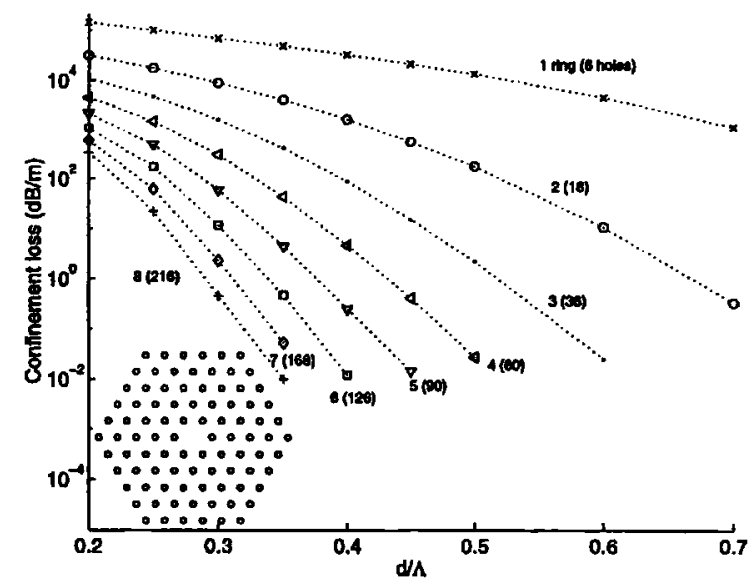

Fig. 3. Confinement loss (in decibels per meter) for MOF's in silica as a function of the number of hexagonally packed rings and their diameter to spacing ratio $d / \Lambda$, with $\Lambda=$ $2.3 \mu \mathrm{m}$, for a wavelength $\lambda=1.55 \mu \mathrm{m}$. Inset, five-ring, $d / \Lambda=0.3$ structure. 


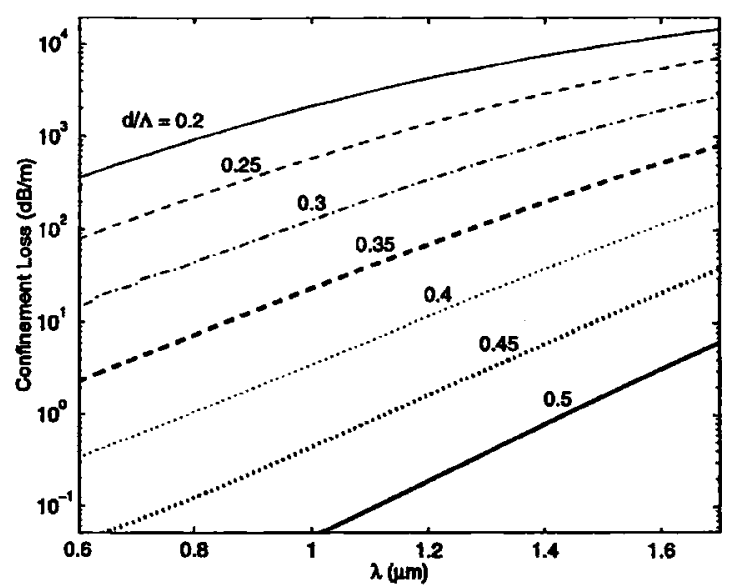

Fig. 4. Confinement loss (in decibels per meter) as a function of wavelength for a MOF with three rings of holes (thirty-six holes) for various hole sizes. The hole spacing is fixed at $2.3 \mu \mathrm{m}$.

$d / \Lambda$, with $\Lambda=2.3 \mu \mathrm{m}$. The silica refractive index again follows from the Sellmeier equation, ${ }^{10}$ and the full material dispersion is thus included. The loss increases smoothly with wavelength, with no evidence of any critical physical dimensions changing the physics of the energy leakage. This increase of loss is consistent with the decrease of $N_{r}$ with wavelength in Fig. 2, implying that the light is less well confined. The same effect occurs in $W$ fibers, which also do not support rigorously bound modes. ${ }^{12}$ From Fig. 4 we note that the losses in Fig. 3 decrease if, at fixed wavelength, $d$ and $\Lambda$ increase while they preserve their ratio.

Loss measurements of MOFs were reported previously. ${ }^{13,14}$ Our results are consistent with those of Windeler et al. in that the losses that they measured ${ }^{13}$ are larger than those indicated by our method. Loss results were also reported by Bennett et al..$^{14}$ for a MOF with three or four rings of small holes that is surrounded by air. In these structures the outer air-glass interface appears to play an important role in confining the light, and a comparison with the results here is not appropriate.

The minimum loss level that we can calculate is determined by machine precision, and, with doubleprecision arithmetic, corresponds to $N_{i} \approx 5 \times 10^{-11}$, or roughly $2 \times 10^{-3} \mathrm{~dB} / \mathrm{m}$ at $\lambda=1.55 \mu \mathrm{m}$ (although the results presented here do not reach this level). As noted, confinement losses can also be calculated by use of the general-purpose vector BPM technique. In contrast, our multipole technique is extremely accurate and efficient for circular inclusions. The BPM is also not well suited for calculating the loss of low-loss modes, as the required propagation distance scales with the length over which the losses are significant.

In conclusion, we have presented a full vector method for MOF calculations that differs significantly from existing methods ${ }^{2-5}$ in that it exploits the circular symmetry of the holes and does not use periodic boundary conditions or a numerical grid. This method leads to an efficient code (all results presented here were obtained with a Compaq Alpha workstation) that allows us to deal with finite systems of holes. It is this feature that lets us calculate confinement losses. The method is also well suited for structures with metallic inclusions, for which traditional methods have trouble coping with the boundary conditions at the interfaces.

The authors thank Tanya Monro for useful discussions during this research, which was supported by the Australian Research Council. R. C. McPhedran's e-mail address is ross@physics.usyd.edu.au.

\section{References}

1. J. C. Knight, T. A. Birks, P. St. J. Russell, and D. M. Atkin, Opt. Lett. 21, 1547 (1996).

2. J. K. Ranka, R. S. Windeler, and A. J. Stentz, Opt. Lett. 25, $796(2000)$.

3. J. Broeng, S. E. Barkou, T. Søndergaard, and A. Bjarklev, Opt. Lett. 25, 96 (2000).

4. D. Mogilevtsev, T. A. Birks, and P. St. J. Russell, J. Lightwave Technol. 17, 2078 (2000).

5. T. M. Monro, D. J. Richardson, N. G. R. Broderick, and P. J. Bennett, J. Lightwave Technol. 17, 1093 (2000).

6. M. J. Steel, T. P. White, C. M. de Sterke, R. C. McPhedran, and L. C. Botten, Opt. Lett. 26, 488 (2001).

7. K. M. Lo, R. C. McPhedran, I. M. Bassett, and G. W. Milton, J. Lightwave Technol. 12, 396 (1994).

8. T. P. White, B. T. Kuhlmey, R. C. McPhedran, D. Maystre, G. Renversez, C. Martijn de Sterke, and L. C. Botten, "Multipole formulation for propagation and loss in microstructured optical fibers," to be submitted to J. Lightwave Technol.

9. M. Abramowitz and I. A. Stegun, Handbook of Mathematical Functions (Dover, New York, 1965).

10. G. P. Agrawal, Nonlinear Fiber Optics (Academic, San Diego, Calif., 1995).

11. J. C. Knight, T. A. Birks, and P. St. J. Russell, J. Opt. Soc. Am. A 15, 748 (1998).

12. L. G. Cohen, D. Marcuse, and W. L. Mammel, IEEE J. Quantum Electron. QE-18, 1467 (1982).

13. R. S. Windeler, J. L. Wagener, and D. J. DiGiovanni, in Optical Fiber Communication Conference (OFC), 1999 OSA Technical Digest Series (Optical Society of America, Washington, D.C., 1999), paper FG1.

14. P. J. Bennett, T. M. Monro, and D. J. Richardson, Opt. Lett. 24, 1203 (1999). 